\title{
Ab Initio and DFT Study of the Conformational Energy Hypersurface of Cyclic Gly-Gly-Gly
}

\author{
Rodrigo D. Tosso, ${ }^{\dagger}$ Miguel A. Zamora, ${ }^{\dagger, \dagger}$ Fernando D. Suvire, ${ }^{\dagger, \ddagger}$ and Ricardo D. Enriz ${ }^{*, \dagger, \sharp}$ \\ Departamento de Química, Facultad de Química, Bioquímica y Farmacia, Universidad Nacional de San Luis, \\ Chacabuco 915, 5700 San Luis, Argentina, and IMIBIO-CONICET, Universidad Nacional de San Luis, \\ Chacabuco 915, 5700 San Luis, Argentina
}

Received: June 2, 2009; Revised Manuscript Received: July 30, 2009

\begin{abstract}
The multidimensional conformational potential energy hypersurface (PEHS) of cyclic Gly-Gly-Gly (1,4,7triazonane-2,5,8-trione) was comprehensively investigated at the Hartree-Fock (RHF/6-31G(d)) level of theory. The equilibrium structures, their relative stability, and the transition state (TS) structures involved in the conformational interconversion pathways were analyzed. aug-cc-pVTZ//B3LYP/6-311++G** single point calculations predict a trans-cis-cis conformation as the energetically preferred form for this compound. However, all of the levels of theory employed here predicted that two forms, a trans-cis-cis and a cis-cis-cis (crown), of conformers contribute significantly to the equilibrium mixture at room temperature. The conformational interconversion between the global minimum and the symmetric cis-cis-cis crown form requires $12.49 \mathrm{kcal} / \mathrm{mol}$ at the RHF 6-31G(d) level of theory, whereas the conformational interconversion between the cis-cis-cis crown and cis-cis-cis boat form requires $18.70 \mathrm{kcal} / \mathrm{mol}$. An exploratory topological analysis of the PEHS was also carried out. Our results allow us to form a concise idea about the internal intricacies of the PEHSs of these cyclic tripeptides, describing the conformations as well as the conformational interconversion processes in these hypersurfaces.
\end{abstract}

\section{Introduction}

There is currently great interest in cyclic peptides and cyclic pseudopeptides (pseudopeptides have been defined as peptides with one or more amide bond replacement, referred to as amide bond surrogates ${ }^{1}$ ). In contrast to linear peptides, cyclic peptides are not susceptible to attack by the exopeptidases (amino- and carboxy-peptidases). Furthermore, their constrained nature reduces the entropic penalty and offers the potential for improved binding, and often improved selectivity, toward a given biological receptor. These properties render cyclic peptides more like peptidomimetics in the continuum between peptides and no peptides in the perpetual search for potent, orally active, and selective drugs. It is clear that a better understanding about the conformational behavior of cyclic peptides is of paramount importance. However, until now, in most cases, only partial solutions might be proposed. This is not an unexpected result; after all, why do we expect a full understanding about the conformational behavior of a complex cyclic structure when many conformational aspects of a very simple cyclic compound like cyclic Gly-Gly-Gly (1,4,7-triazonane-2,5,8-trione) remain unknown? It is clear that the elucidation of more details about the conformational behavior of small-size cyclic peptides could help us to better understand the conformational behavior of medium- and large-size cyclic compounds of biological interest.

Special attention must be devoted to small-size cyclic peptides, since conformational searching in such systems is generally more intricate than for the large-size congeners. It is not always easy to discriminate how many independent torsion

* To whom correspondence should be addressed. Phone: +542652 424689, extension 157. Fax: +54 2652 431301. E-mail: denriz@unsl.edu.ar.

${ }^{\dagger}$ Departamento de Química, Facultad de Química, Bioquímica y Farmacia.

* IMIBIO-CONICET. angles must be varied to go from one conformer to another one. The deformation of rings via bond rotation is a difficult problem, since the dihedral angles are not independent variables. Thus, small-size cyclic peptides form an interesting class of compounds for study by conformational analysis by virtue of their unique conformational features and biological properties. However, exploration of conformational space is a difficult problem, which is especially acute for cyclic molecules due to the interdependence of torsional angles. ${ }^{2}$ For small-size cyclic systems, the potential energy hypersurface (PEHS) may have a small number of local minima as well as transition states interconnecting these minima. Nevertheless, to determine all of the critical points is not always an easy task ${ }^{3}$ in particular if we are optimizing these structures at a relatively high level of theory. Furthermore, finding the global minimum energy conformation alone may not be sufficient. In general, it is necessary to locate all of the critical points one can possibly find on an energy hypersurface first and then to examine their stability and their ease of conversion to other conformers, determining their relative importance. It is clear that this is a problem of considerable computational magnitude when partially flexible rings are involved. Previously, we reported a comprehensive conformational study of the PEHS of cyclononane using $a b$ initio and DFT calculations. ${ }^{4}$ Our results showed that the PEHS of this apparently simple molecule in fact is very complex.

Cyclic tripeptides which contain nine-member rings are in general strained systems. Not many cyclic tripeptides are known. Those on which crystal structure information is available are cyclo trisarcosyl ${ }^{5}$ and others containing Prolyl and $N$-BzlGlycyl residues. One common feature in all of these is the occurrence of $c i s$ peptide units which is geometrically a necessary factor for the formation of cyclic tripeptides. $N$-Methylated cyclic triglycine has a $C_{3}$ symmetry conformation (crown form) 

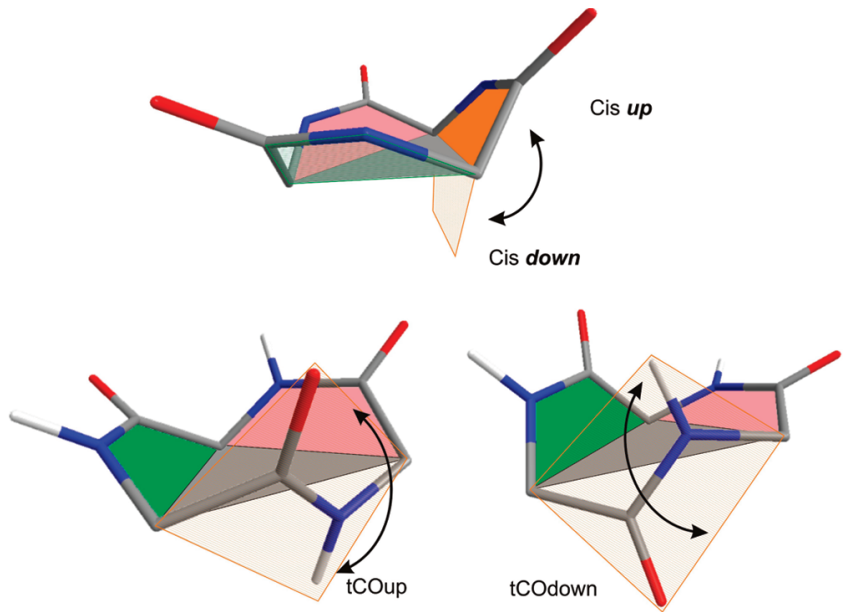

Figure 1. Schematic view of CTG showing in gray the central plane determined by the three methylene groups ( $\alpha$ carbons) and in different colors the three planes determined by each peptide bond. In the upper zone, the change from up to down of the cis peptide plane is shown. In the bottom, the change from tCOup to tCOdown of the trans peptide plane is shown.

elucidated by $\mathrm{NMR}^{6,7}$ and X-ray crystallography studies. ${ }^{8}$ Hioki et al. ${ }^{9}$ reported the synthesis of $\mathrm{N}$-substituted cyclic triglycines using $N, N^{\prime}, N^{\prime \prime}$-triallyl-cyclo-triglicine as a scaffold. The ${ }^{1} \mathrm{H}$ and ${ }^{13} \mathrm{C} \mathrm{NMR}$ spectra of this compound indicate that the major conformation was the crown form along with some "saddle" (they called boat) form. Kessler and his group have done extensive studies both in solution and in solid state, on cyclic tripeptides containing NBzlGly and Prolyl residues. ${ }^{10,11}$ While cyclo (BzlGly-L-Pro-L-Pro) ${ }^{12}$ and cyclo $^{13}$ (BzlGly $_{2}$-L-Pro) take up symmetric conformations, cyclo (NitroBzlGly-L-Pro 2 ) assumes the asymmetric conformation in the solid state. ${ }^{14}$ The reason for this is still not clear and needs further study. In fact, there are very few theoretical calculations performed on these cyclic tripeptides and, also, in general these computations have been carried out using low levels of theory. To the best of our knowledge, the only conformational study performed for cyclic triglycine was reported by Ramakrishan et al. ${ }^{15}$ using MM calculations. However, it must be pointed out that in that article the complete PEHS of this compound was not evaluated. More recently, we reported the PEHS of three cyclic compounds possessing nine carbon atoms: cis-cis-cis-1,4,7-cyclononatriene, tribenzocyclononatriene, and cis-cis-cis cyclic triglycine $^{5}$ (CTG), but in that article, only the cis-cis-cis form of cyclic triglycine was considered. Our results indicated that the conformational interconversion process in cyclic peptides is somewhat different from those of cyclic polyenes. ${ }^{16}$

In the present paper, we performed a systematic and comprehensive analysis about the different conformational interconversion process for CTG. Thus, we report here an exhaustive conformational analysis of CTG using $a b$ initio and density functional theory (DFT) computations. Aside from the populations of the conformers, it is of great interest to know how the interconversions between the conformers are and which of them occur most readily. The equilibrium structures, their relative stability, and the transition state (TS) structures involved in the conformational interconversion pathways were analyzed. General topological aspects of this PEHS are also discussed in the first section of this paper.

\section{Calculations}

All of the calculations reported here were performed using the Gaussian 03 program. ${ }^{17}$ Critical points (low-energy conformations and transition state structures) were optimized at the RHF/6-31G(d) level of theory. An extensive search to localize the critical points on the PEHSs was carried out first by using starting geometries suggested by the GASCOS algorithm. ${ }^{18-21}$ Previously, we reported a systematic search method called geometrical algorithm to search the conformational space (GASCOS) for open chain compounds. ${ }^{18}$ This article is primarily devoted to the mathematical principles on which this approach

TABLE 1: The 12 Different Conformers Obtained for CTG from RHF/6-31G(d) Calculations (The Corresponding Isomer and Topology Type Are Also Shown)

\begin{tabular}{|c|c|c|c|c|}
\hline conformer & isomer & topology & relative energy $(\mathrm{kcal} / \mathrm{mol})$ & entropy $(\mathrm{cal} / \mathrm{mol} \cdot \mathrm{K})$ \\
\hline 1 & trans $-c i s-c i s$ & tCOdown-up-up & 0.00 & 99.120 \\
\hline 2 & cis-cis-cis & up-up-up & 0.71 & 100.541 \\
\hline 3 & cis-cis-cis & down-up-up & 2.86 & 101.533 \\
\hline 5 & trans - cis - cis & tCOup-up-up & 6.10 & 99.948 \\
\hline 6 & trans - cis - cis & tCOup-down-up & 7.02 & 98.477 \\
\hline 7 & trans-trans-trans & tCOup - tCOdown - tCOdown & 7.62 & 95.598 \\
\hline 10 & trans - trans - cis & tCOdown-tCOdown-up & 13.19 & 99.986 \\
\hline 11 & trans-trans-cis & tCOdown-tCOdown-down & 13.47 & 99.205 \\
\hline 12 & trans-trans-trans & tCOdown - tCOdown - tCOdown & 17.08 & 98.460 \\
\hline
\end{tabular}

TABLE 2: Energy Gaps in kcal/mol Obtained for the Three Preferred Conformations of CTG and Their Transition States Using Different Levels of Theory

\begin{tabular}{|c|c|c|c|c|c|c|c|c|c|c|}
\hline \multirow[b]{2}{*}{$\begin{array}{l}\text { conformer/ } \\
\text { TS }\end{array}$} & \multicolumn{2}{|c|}{ RHF/6-31G(d) } & \multicolumn{2}{|c|}{ B3LYP/6-31G(d) } & \multicolumn{2}{|c|}{ B3LYP/6-31G(d,p) } & \multicolumn{2}{|c|}{ B3LYP/6-311++G(d,p) } & $\begin{array}{c}\text { MP2/6-31G(d,p)//B3LYP/ } \\
6-311++\mathrm{G}(\mathrm{d}, \mathrm{p})\end{array}$ & $\begin{array}{c}\text { B3LYP/Aug-cc-pvtz// } \\
\text { B3LYP/6-311++G(d,p) }\end{array}$ \\
\hline & $\Delta E$ & $\begin{array}{l}\text { imaginary } \\
\text { frequency }\end{array}$ & $\Delta E$ & $\begin{array}{l}\text { imaginary } \\
\text { frequency }\end{array}$ & $\Delta E$ & $\begin{array}{l}\text { imaginary } \\
\text { frequency }\end{array}$ & $\Delta E$ & $\begin{array}{l}\text { imaginary } \\
\text { frequency }\end{array}$ & $\Delta E$ & $\Delta E$ \\
\hline 1 & 0.00 & & 0.00 & & 0.00 & & 0.58 & & 0.00 & 1.09 \\
\hline 2 & 0.71 & & 0.29 & & 0.43 & & 0.00 & & 1.72 & 0.00 \\
\hline 3 & 2.86 & & 2.99 & & 2.97 & & 2.68 & & 3.74 & 3.17 \\
\hline $\mathbf{T S}_{1-2}$ & 12.49 & -129.883 & 16.66 & -220.422 & 16.56 & -213.565 & 16.26 & -229.220 & 15.31 & 16.70 \\
\hline $\mathbf{T S}_{1-3}$ & 20.42 & -289.212 & 22.79 & -379.529 & 22.63 & -405.431 & 22.35 & -416.060 & 22.24 & 22.57 \\
\hline $\mathbf{T S}_{2-3}$ & 18.70 & -200.093 & 17.44 & -182.957 & 17.53 & -183.990 & 17.81 & -190.651 & 18.98 & 17.94 \\
\hline
\end{tabular}




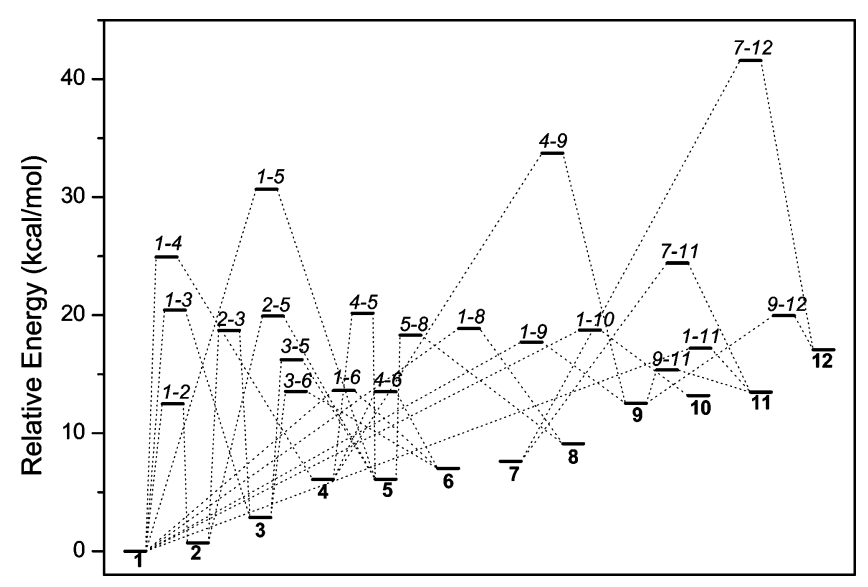

Figure 2. Energy profiles in kcal/mol obtained from RHF/6-31G(d) calculations for the different diasteromeric interconversions. The minima are denoted in bold and the transition states in italic.

is based. GASCOS is able to assess the spatial requirement and, therefore, spatial proximity of rotating moieties within a given molecule. It also produced useful input data for energy calculating programs (e.g., ab initio MO). In fact, GASCOS may be converted to be used as an automatic driver program for exploring multidimensional conformational space. We extended the capability of this algorithm for the study of conformationally flexible cyclic molecules. ${ }^{19,20}$ The GASCOS algorithm possesses a novel analytical ring closure constraint, which improved the performance of this search technique. The method is readily applied as a tree search, which makes feasible analyses of cyclic molecules containing many internal degrees of freedom, for example, several dihedral angles. Next, a matrix representation was developed for two successive rotations in order to calculate easily the atomic coordinates from the initial molecular geometry and the dihedral angles applied. ${ }^{21}$ However, it should be noted that this matrix representation would be more useful in the case of noncyclic compounds where the dihedral angles can take a predetermined combination of values.

Input files obtained from GASCOS were used to obtain the low-energy conformations and TS structures using RHF/6$31 \mathrm{G}(\mathrm{d})$ calculations. Vibrational frequencies for the optimized structures were computed to evaluate the zero-point energies (ZPEs) as well as to confirm the nature of the singular points along the potential energy surface. The stationary points have been identified as a minimum with no imaginary frequencies, or as a first-order transition state characterized by the existence of only one imaginary frequency in the normal mode coordinate analysis. Transition state structures were located until the Hessian matrix had only one imaginary eigenvalue, and the transition states were also confirmed by animating the negative eigenvector coordinate with a visualization program and internal reaction coordinate (IRC) calculations. ${ }^{22,23} \mathrm{HF} / 6-31 \mathrm{G}$ (d) IRC calculations were performed on the transition state structures to check that the TS structures lead to the initial conformer and to the final conformation (forward and reverse directions of the conformational interconversion path). IRC calculations were carried out along the path in Cartesian coordinates in six points forward and six points in the reverse direction, in steps of 03 $\mathrm{amu}^{1 / 2}$ bohr.

After obtaining the optimized structures from RHF/6-31G(d) calculations, single point calculations using the most reliable and flexible basis set (aug-cc-pVTZ) were carried out in order to evaluate the energies of the preferred conformers.

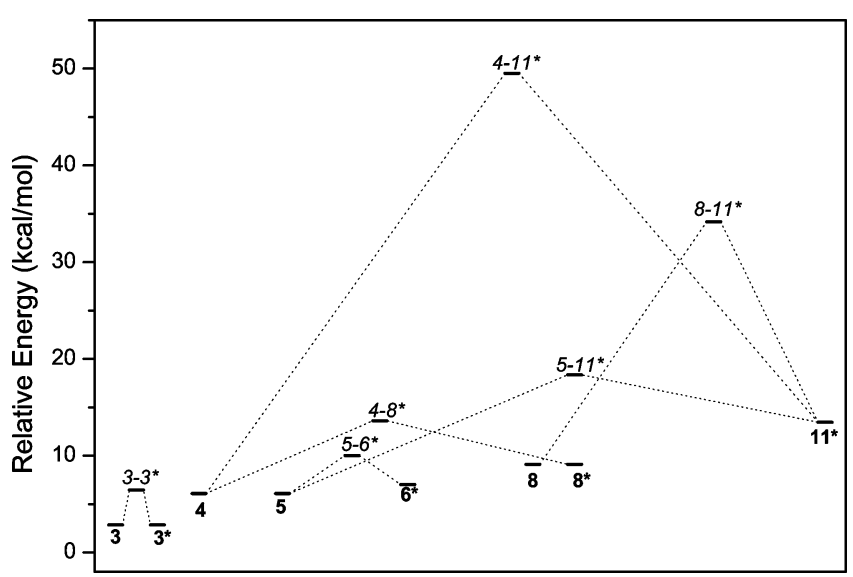

Figure 3. Energy profiles in $\mathrm{kcal} / \mathrm{mol}$ obtained from $\mathrm{RHF} / 6-31 \mathrm{G}(\mathrm{d})$ calculations for the different enantiomeric interconversions. The minima are denoted in bold and the transition states in italic.

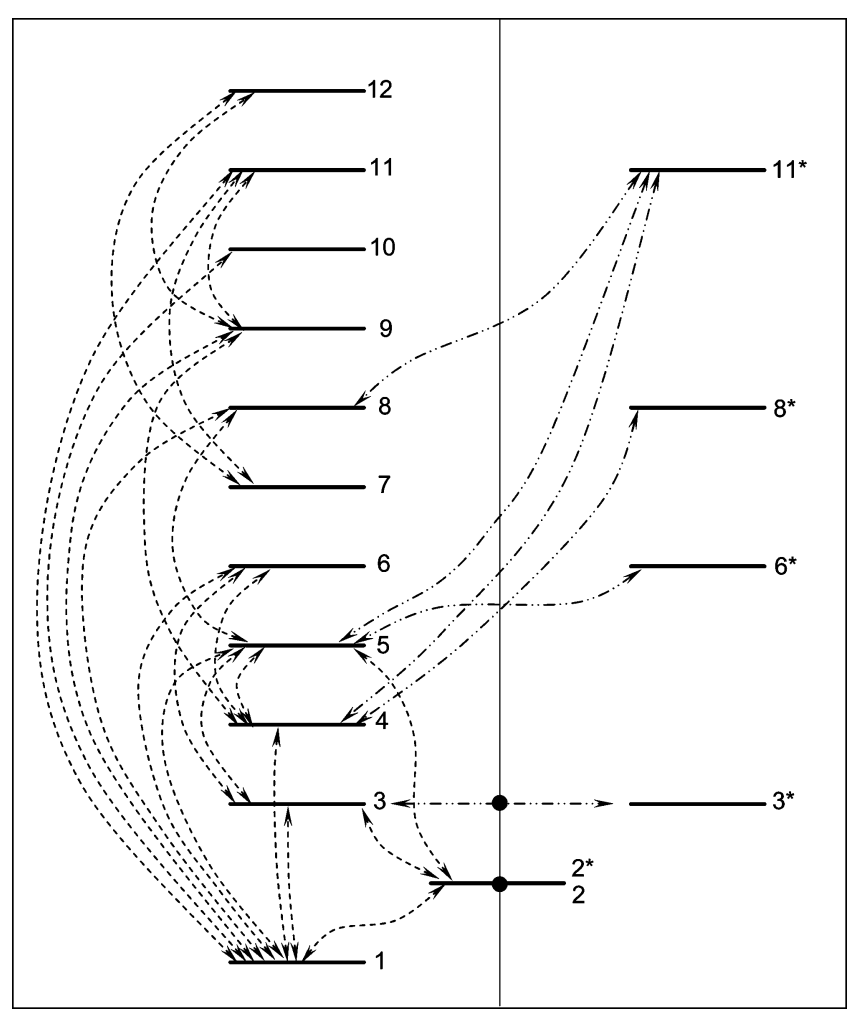

Figure 4. Schematic diagram showing diasteromeric (dashed line) and enantiomeric (dash - dot - dot line) interconversions. The two symmetric forms $\mathbf{2}$ and $\mathbf{T S}_{3_{-3}}$ are denoted by a bold dot. The central line represents a mirror separating the specular images.

\section{Results and Discussion}

Exploratory Topological Analysis of the HSEP. In a first step of our study, we performed an exploratory topological analysis of the HSEP of CTG. Although mathematically irrelevant, the problem of visualization of hypersurfaces is of considerable interest because it makes it possible to rationalize the conformational behavior of cyclic compounds. In the case of flexible cyclic peptides, it is extremely difficult to derive proper qualitative conclusions by merely inspecting a manifold of energy values and coordinates. Probably, the most convenient way to visualize the hypersurface of CTG is to define some relevant structural elements such as characteristic planes and their respective spatial orderings. From the structural characteristics of CTG, two different types of planes can be considered: (a) a central plane determined by the three methylene groups 
TABLE 3: The 27 TS Structures Obtained for the Different Conformational Interconversions Observed in CTG

\begin{tabular}{|c|c|c|c|}
\hline $\begin{array}{l}\text { transition } \\
\text { state }\end{array}$ & type & relative energy (kcal/mol) & $\begin{array}{l}\text { imaginary } \\
\text { frequencies }\end{array}$ \\
\hline $3-3^{*}$ & A & 6.46 & -102.251 \\
\hline $5-6 *$ & $\mathrm{E}$ & 10.03 & -130.715 \\
\hline $1-2$ & $\mathrm{Fb}$ & 12.49 & -129.883 \\
\hline $4-6$ & $\mathrm{Fb}$ & 13.51 & -72.482 \\
\hline $3-6$ & $\mathrm{Fb}$ & 13.55 & -119.382 \\
\hline $1-6$ & $\mathrm{E}$ & 13.61 & -148.482 \\
\hline $4-8 *$ & $\mathrm{E}$ & 13.62 & -160.624 \\
\hline $9-11$ & $\mathrm{E}$ & 15.36 & -123.945 \\
\hline $3-5$ & $\mathrm{~F}$ & 16.22 & -199.582 \\
\hline $1-11$ & $\mathrm{Fb}$ & 17.20 & -148.351 \\
\hline $1-9$ & $\mathrm{Fb}$ & 17.71 & -156.005 \\
\hline $5-8$ & $\mathrm{~F}$ & 18.31 & -188.632 \\
\hline $5-11 *$ & B & 18.36 & -407.879 \\
\hline $2-3$ & $\mathrm{E}$ & 18.70 & -200.093 \\
\hline $1-10$ & $\mathrm{~F}$ & 18.73 & -178.501 \\
\hline $1-8$ & B & 18.87 & -500.271 \\
\hline $2-5$ & $\mathrm{~F}$ & 19.92 & -220.846 \\
\hline $9-12$ & B & 19.96 & -256.202 \\
\hline $4-5$ & $\mathrm{Fb}$ & 20.16 & -163.844 \\
\hline $1-3$ & B & 20.42 & -289.212 \\
\hline $7-11$ & $\mathrm{D}$ & 24.42 & -117.593 \\
\hline $1-4$ & $\mathrm{~F}$ & 24.93 & -167.097 \\
\hline $1-5$ & $\mathrm{C}$ & 30.68 & -358.471 \\
\hline 4-9 & $\mathrm{C}$ & 33.72 & -260.530 \\
\hline $8-11 *$ & $\mathrm{C}$ & 34.17 & -481.658 \\
\hline $7-12$ & $\mathrm{C}$ & 41.56 & -285.180 \\
\hline $4-11 *$ & $\mathrm{C}$ & 49.50 & -286.096 \\
\hline
\end{tabular}

( $\alpha$ carbons) and (b) the three planes determined by each peptide bond, which can be located in two different forms depending on the cis or trans arrangement of such peptide bond (Figure 1).

Taking the central plane as a reference, we considered the relative movements determined by the different spatial orderings of the other three planes. Thus, there are only two possibilities of cis-cis-cis arrangement: (a) when the three peptide-bond planes are in the same position (up-up-up or down-downdown), giving the two enantiomeric crown forms ( 2 and $2 *$ ), and (b) when only two peptide-bond planes are located in the same position, which gives the so-called twist forms (up-updown or down-down-up). There are six additional forms related to these; however, in CTG, these are degenerated and nondistinguishable structures. For simplicity, in this study, we consider only one typical form for each case. In the transcis-cis arrangement, four different structures can be observed: when the two cis planes are located in the same position with respect to the central plane or when they are located in opposite arrangements. In addition, the trans-peptide plane can adopt two different forms: with the carbonyl group up (tCOup) or down (tCOdown). Therefore, the trans - cis-cis arrangement gives four different forms (Figure 1). The trans-trans-cis arrangement in turn possesses a cis-peptide plane which can be up or down and two trans-peptide planes which can also be located up or down. Thus, the combinations give four general orderings (Table 1). This case is closely related to the previous one. The trans-trans-trans form can give only two different possibilities: with the carbonyl groups located in the same side or in opposite positions. It is clear that this is a very simple analysis; however, it can be useful to obtain a picture about the number of potentially different conformers in the PEHS.

Computation and Analysis of Critical Points on the PEHS of Cyclic Tryglicine. Considering the putative 12 different forms obtained from the exploratory topological analysis as starting geometries, RHF/6-31G(d) optimizations gave 12 different conformations on the PEHS of CTG. These results are summarized in Table 1. It should be noted that only 11 different forms out of the hypothetical 12 forms described in the previous section were obtained, and that no conformer was found possessing a tCOdown-down-up arrangement. In contrast, two different conformers were found displaying the tCOdowntCOdown-up arrangement. Thus, we obtained two conformations (2 and $\mathbf{3}$ ) for the cis-cis-cis form, three conformations $(\mathbf{1}, \mathbf{5}$, and $\mathbf{6})$ possessing the trans - cis-cis form, five conformations $(4,8,9,10$, and 11) displaying the trans-trans-cis form, and two conformers (7 and 12) with the trans-trans-trans arrangement (Table 1). RHF/6-31G(d) calculations predict that the energetically preferred form is conformation $\mathbf{1}$ possessing a trans-cis-cis form, whereas the crown form (conformation 2) is the lowest-energy local minimum with an energy gap of $0.71 \mathrm{kcal} / \mathrm{mol}$ above the global minimum. The twist form (conformation 3, also called boat ${ }^{9}$ ) is the third preferred form with an energy gap of $2.86 \mathrm{kcal} / \mathrm{mol}$. Conformations $\mathbf{1}, \mathbf{2}$, and $\mathbf{3}$, or at least very similar forms, have been previously reported by Ramakrishan et al. ${ }^{15}$ from molecular mechanic calculations. However, it is interesting to note that Ramakrishan reported the crown form as the highly preferred conformation. In the presence of such a situation, a reasonable step to take is to look for an arbitrage by a still more refined procedure. Thus, these three conformations and their respective transition states were optimized using different levels of theory: B3LYP/6-31G(d), B3LYP/6-31G(d,p), and B3LYP/6-311++G(d,p). In addition, single point calculations using the more flexible basis set augcc-pVTZ and MP2/6-31G(d,p) computations were carried out in order to evaluate the energies of these three conformers and their transition states (see Table 2). All of the calculations reported here indicate that forms $\mathbf{1}$ and $\mathbf{2}$ are in an almost perfect equilibrium mixture of conformations. Thus, on the basis of our results, both forms $\mathbf{1}$ and $\mathbf{2}$ can be expected to be significantly populated. Regarding the rest of the conformations, only conformation 3 possessing $2.86 \mathrm{kcal} / \mathrm{mol}$ above the global minimum (at the RHF/6-31G(d) level of theory) should contribute somewhat to the equilibrium mixture of conformations. The energy gaps obtained for conformations 4, 5, and $\mathbf{6}$ are $6.09,6.10$, and $7.02 \mathrm{kcal} / \mathrm{mol}$, respectively; therefore, none of them is expected to contribute significantly to the equilibrium mixture at room temperature.

In contrast to the results obtained for CTG, our preliminary calculations performed for cyclic trisarcosyl indicate a crown conformation as the energetically preferred form for this molecule (data not shown). These results are in complete agreement with the experimental data obtained from X-ray ${ }^{5}$ and $\mathrm{NMR}^{7}$ results.

Progress in searching for transition states and their significance is likely to be much more demanding in computer time than simply a search for local energy minima, because just locating the saddle points on the PEHS is not sufficient. It is also necessary to find out how the low-energy minima and the TSs are linked together, which requires an exploration of a larger part of the hypersurface. CTG has 12 low-energy conformations on its PEHSs, but it is necessary to know where one form changes to the other and how far from an energy minimum the molecule can stray away before ceasing to be in the other conformation. It is clear, therefore, that information about the local and the global minimum of molecules such as CTG is not enough. We need to have an understanding of its shape and also some indication of the dynamic behavior of the internal degrees of freedom of these compounds. A full understanding 


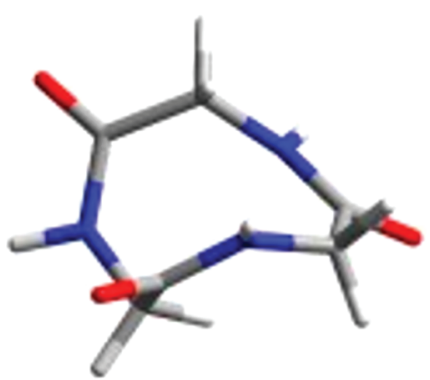

3

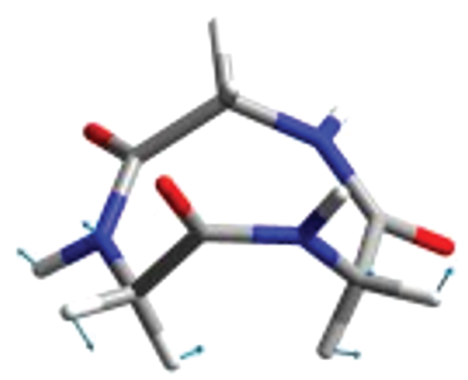

$\mathbf{T S}_{3-3^{*}}$

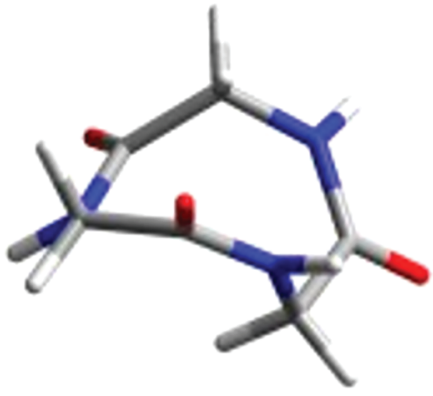

$3 *$

Figure 5. TS type A. Optimized structures of low-energy forms and transition state at the RHF/6-31G(d) level of theory. The result of the vibrational analysis of the transition state is also shown; the length of each arrow is proportional to the degree of vibration of the atom.

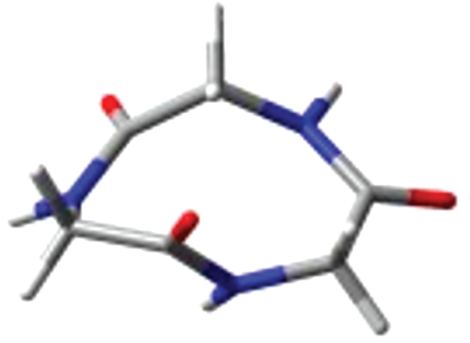

1

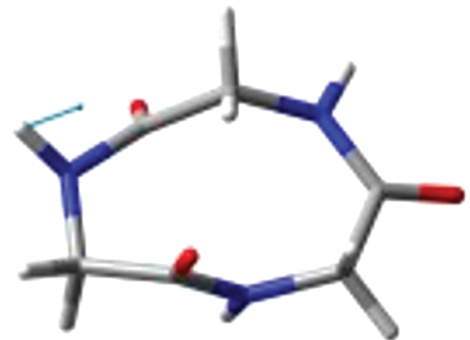

$\mathbf{T S}_{1-8}$

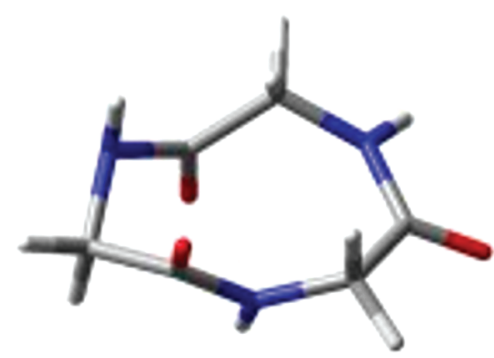

8

Figure 6. TS type B. Optimized structures of low-energy forms and transition state at the RHF/6-31G(d) level of theory. The result of the vibrational analysis of the transition state is also shown; the length of each arrow is proportional to the degree of vibration of the atom.

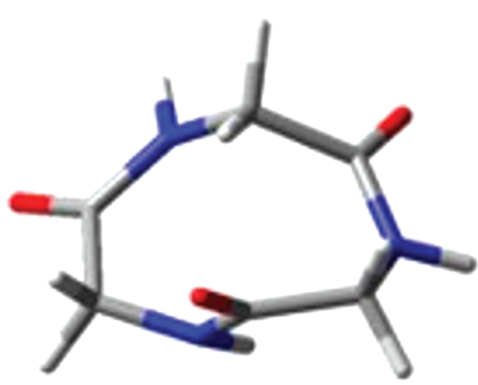

1

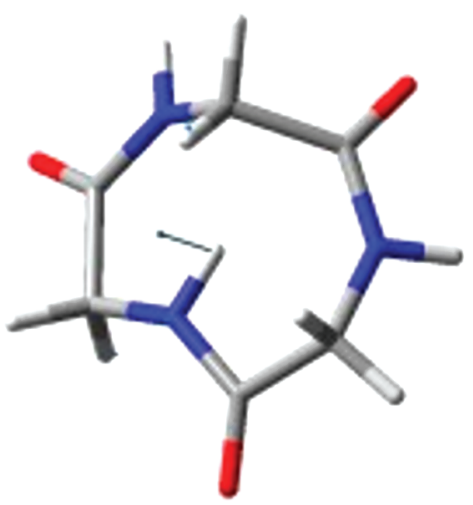

$\mathrm{TS}_{1-5}$

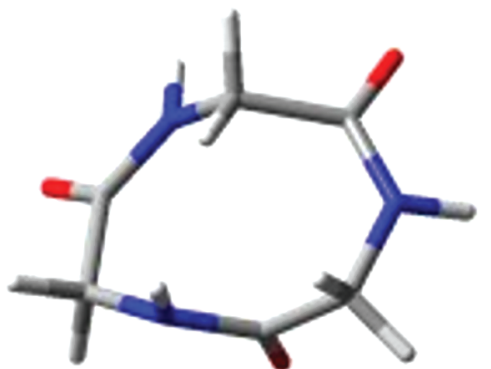

5

Figure 7. TS type C. Optimized structures of low-energy forms and transition state at the RHF/6-31G(d) level of theory. The result of the vibrational analysis of the transition state is also shown; the length of each arrow is proportional to the degree of vibration of the atom.

of the PEHS requires identification of the complete network of conformational interconversions among the different critical points. The GASCOS algorithm combined with RHF/6-31G(d) optimizations found 27 different transition states on the PEHS of CTG (see Figures 2 and 3). However, it should be noted that only 21 of these transition states are connecting nonenantiomeric forms (Figure 2), whereas 6 structures are connecting nonenantiomeric forms with enantiomeric ones (Figure 3 ). The enantiomeric forms are denoted by stars in this figure.

Due to the symmetric characteristics of CTG, there is a set of enantiomeric forms for each conformation. The conformational interconversion between each set of conformers can occur through symmetric structures (conformers such as $\mathbf{2}$ or transition states connecting mirror images such as $\left.\mathbf{T S}_{\mathbf{3}-\mathbf{3}^{*}}\right)$ as well as nonsymmetric structures (transition states connecting mirror forms of different conformations such as all of the remaining TSs shown in Figure 3). This situation can be clearly seen from Figure 4 which shows the different conformational interconversions obtained in the PEHS of CTG. The different types of conformational interconversions are discussed in detail in the next section.

Different Steps in the Conformational Interconversion Paths. Six different conformational interconversion paths were

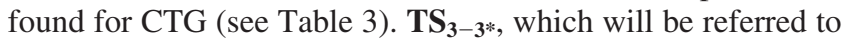
as type A, is a relatively symmetric structure which displays a conrotatory movement for the two methylene groups. Only one 


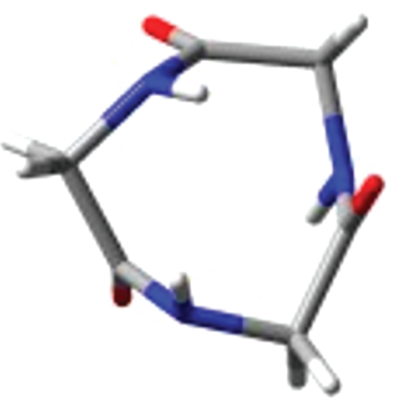

7

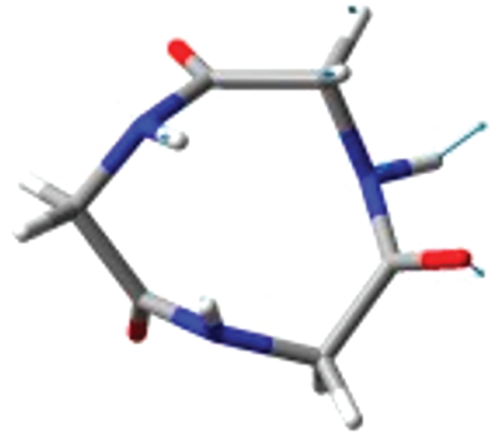

$\mathbf{T S}_{7-11}$

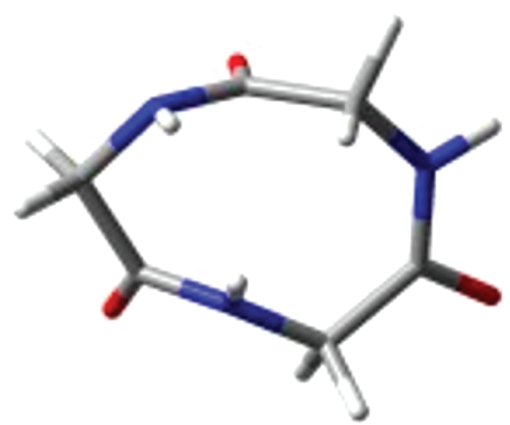

11

Figure 8. TS type D. Optimized structures of low-energy forms and transition state at the RHF/6-31G(d) level of theory. The result of the vibrational analysis of the transition state is also shown; the length of each arrow is proportional to the degree of vibration of the atom.

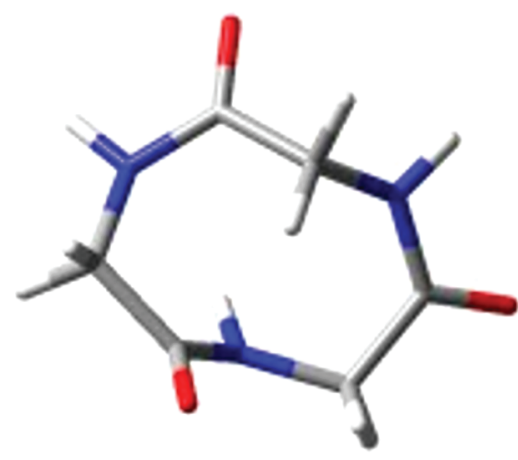

1

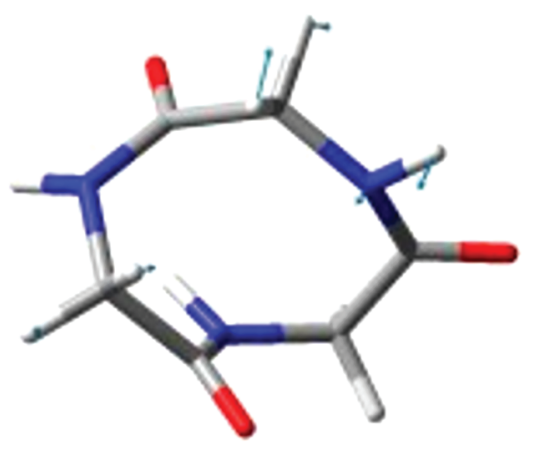

$\mathrm{TS}_{\text {1-6 }}$

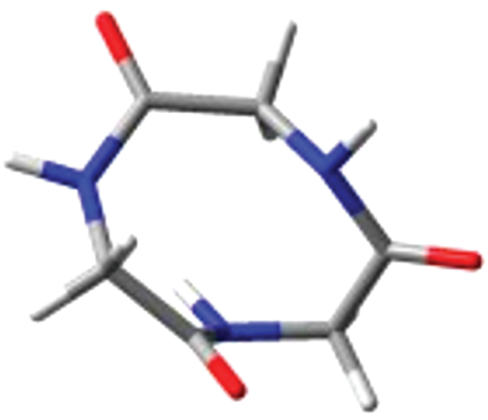

6

Figure 9. TS type E. Optimized structures of low-energy forms and transition state at the RHF/6-31G(d) level of theory. The result of the vibrational analysis of the transition state is also shown; the length of each arrow is proportional to the degree of vibration of the atom.

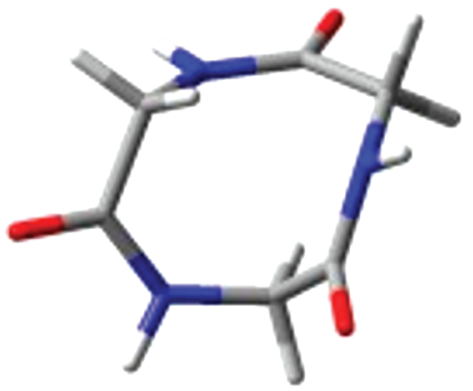

3

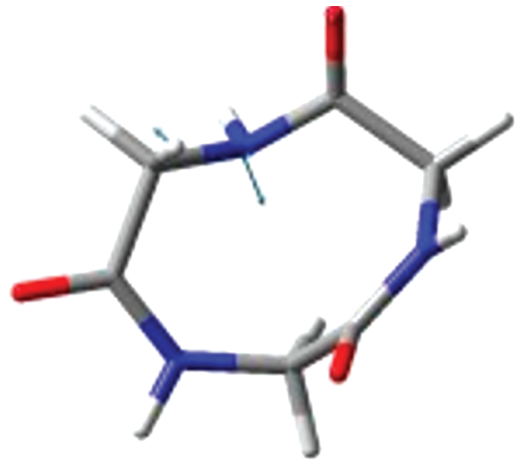

$\mathrm{TS}_{3-5}$

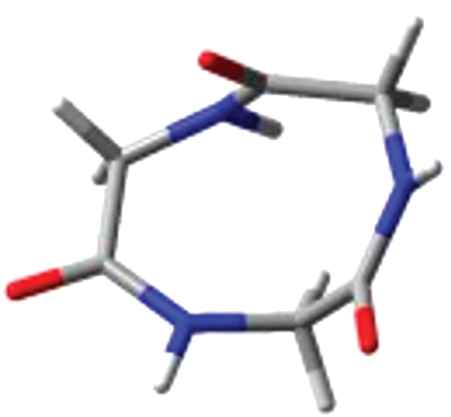

5

Figure 10. TS type F. Optimized structures of low-energy forms and transition state at the RHF/6-31G(d) level of theory. The result of the vibrational analysis of the transition state is also shown; the length of each arrow is proportional to the degree of vibration of the atom.

TS of type A was found. It should be noted that this transition structure possesses very low energy because it involves a very simple and fast spatial rearrangement displaying only significant changes in the affected torsional angles. Figure 5 gives a spatial view of this conformational interconversion process.

The second class of TS, which will be referred to as type B, is shown in Figure 6. $\mathbf{T S}_{\mathbf{1 - 8}}$ connects the global minimum with conformer 8 . This conformational interconversion involves the change of a peptide bond from cis to trans. The most important movement here is carried out by the $\mathrm{N}-\mathrm{H}$ group performing a pyramidal inversion and the consequent rearrangement. We found four TSs displaying this conformational interconversion
$\left(\mathbf{T S}_{\mathbf{1 - 8}}, \mathbf{T S}_{\mathbf{5 - 1 1 ^ { * }}}, \mathbf{T S}_{\mathbf{9}_{-\mathbf{1 2}} \text {, and }} \mathbf{T S}_{\mathbf{1 - 3}}\right)$. The energy requirement for these processes varies from 18.36 to $20.42 \mathrm{kcal} / \mathrm{mol}$.

A third type of interconversion, type $\mathrm{C}$, connects conformers possessing the same configuration and involves the change of the trans-peptide bond. This change is mainly driven by the "switch" of the NH group from planar to pyramidal. This change involves the internal crossing of the $\mathrm{H}$ atom throughout the ring giving the spatial inversion of the trans-peptide bond (Figure 7). The proximity of the van der Waals radii when the $\mathrm{H}$ atom is crossing inside the ring is probably responsible for the high energy required for this conformational interconversion (from 


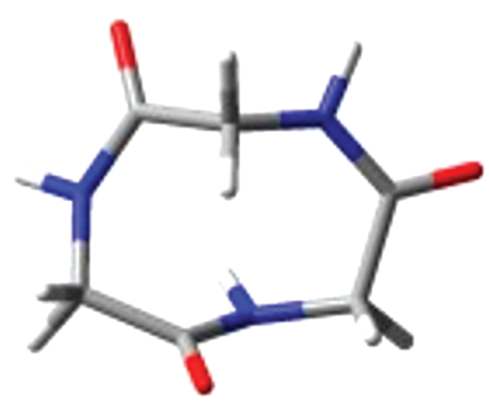

1

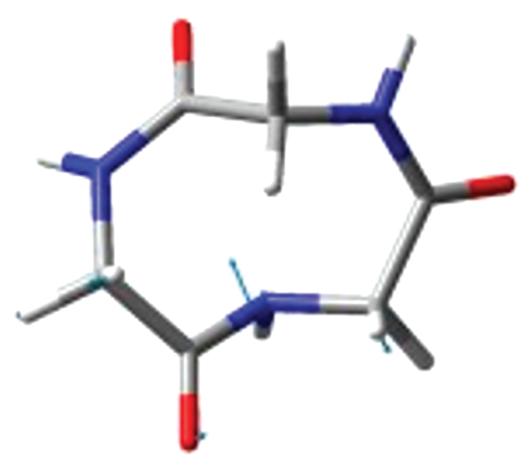

$\mathrm{TS}_{1-2}$

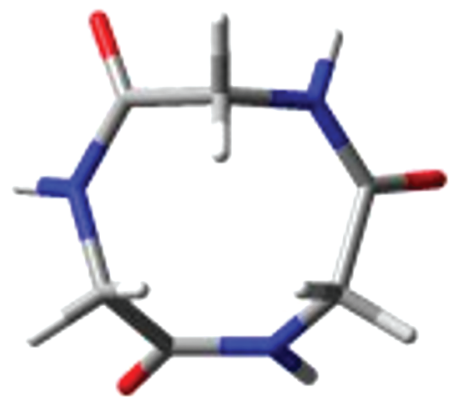

2

Figure 11. TS type Fb. Optimized structures of low-energy forms and transition state at the RHF/6-31G(d) level of theory. The result of the vibrational analysis of the transition state is also shown; the length of each arrow is proportional to the degree of vibration of the atom.

TABLE 4: The Specific Constant of Velocity for Each Conformational Interconversion Using the Eyring Formulation (The $\Delta H$ and $\Delta S$ Values Are Also Shown)

\begin{tabular}{lrrl}
\hline transition & $\Delta H(\mathrm{kcal} / \mathrm{mol})$ & $\Delta S(\mathrm{cal} / \mathrm{mol} \cdot \mathrm{K})$ & \multicolumn{1}{c}{} \\
\hline $\mathbf{2} \rightarrow \mathbf{1}$ & 11.78 & -4.11 & $1.80 \times 10^{3}$ \\
$\mathbf{3} \rightarrow \mathbf{1}$ & 17.56 & -2.61 & $2.20 \times 10^{-1}$ \\
$\mathbf{4} \rightarrow \mathbf{1}$ & 18.84 & -0.33 & $7.99 \times 10^{-2}$ \\
$\mathbf{5} \rightarrow \mathbf{1}$ & 24.58 & -1.88 & $2.26 \times 10^{-6}$ \\
$\mathbf{6} \rightarrow \mathbf{1}$ & 6.59 & -2.18 & $3.04 \times 10^{7}$ \\
$\mathbf{8} \rightarrow \mathbf{1}$ & 9.76 & 0.45 & $5.41 \times 10^{5}$ \\
$\mathbf{9} \rightarrow \mathbf{1}$ & 5.18 & -1.84 & $3.90 \times 10^{8}$ \\
$\mathbf{1 0} \rightarrow \mathbf{1}$ & 5.54 & -4.40 & $5.86 \times 10^{7}$ \\
$\mathbf{1 1} \rightarrow \mathbf{1}$ & 3.73 & -2.92 & $2.62 \times 10^{9}$ \\
$\mathbf{1} \rightarrow \mathbf{2}$ & 12.49 & -2.68 & $1.11 \times 10^{3}$ \\
$\mathbf{1} \rightarrow \mathbf{3}$ & 20.42 & -0.20 & $5.92 \times 10^{-3}$ \\
$\mathbf{1} \rightarrow \mathbf{4}$ & 24.93 & -1.94 & $1.21 \times 10^{-6}$ \\
$\mathbf{1} \rightarrow \mathbf{5}$ & 30.68 & -1.05 & $1.15 \times 10^{-10}$ \\
$\mathbf{1} \rightarrow \mathbf{6}$ & 13.61 & -2.82 & $1.56 \times 10^{2}$ \\
$\mathbf{1} \rightarrow \mathbf{8}$ & 18.87 & 0.43 & $1.11 \times 10^{-1}$ \\
$\mathbf{1} \rightarrow \mathbf{9}$ & 17.71 & -1.73 & $2.66 \times 10^{-1}$ \\
$\mathbf{1} \rightarrow \mathbf{1 0}$ & 18.73 & -3.53 & $1.92 \times 10^{-2}$ \\
$\mathbf{1} \rightarrow \mathbf{1 1}$ & 17.20 & -2.38 & $4.54 \times 10^{-1}$ \\
$\mathbf{3} \rightarrow \mathbf{2}$ & 15.84 & -2.48 & $4.30 \times 10^{0}$ \\
$\mathbf{5} \rightarrow \mathbf{2}$ & 13.82 & -1.05 & $2.67 \times 10^{2}$ \\
$\mathbf{2} \rightarrow \mathbf{3}$ & 17.99 & -1.49 & $1.87 \times 10^{-1}$ \\
$\mathbf{2} \rightarrow \mathbf{5}$ & 19.21 & -1.64 & $2.21 \times 10^{-2}$ \\
$\mathbf{5} \rightarrow \mathbf{3}$ & 10.12 & -1.39 & $1.17 \times 10^{5}$ \\
$\mathbf{6} \rightarrow \mathbf{3}$ & 6.53 & -1.22 & $5.46 \times 10^{7}$ \\
$\mathbf{3} \rightarrow \mathbf{5}$ & 13.36 & -2.97 & $2.21 \times 10^{2}$ \\
$\mathbf{3} \rightarrow \mathbf{6}$ & 10.69 & -4.26 & $1.05 \times 10^{4}$ \\
$\mathbf{5} \rightarrow \mathbf{4}$ & 14.06 & -4.38 & $3.34 \times 10^{1}$ \\
$\mathbf{6} \rightarrow \mathbf{4}$ & 6.49 & -3.67 & $1.70 \times 10^{7}$ \\
$\mathbf{9} \rightarrow \mathbf{4}$ & 21.19 & -3.41 & $3.21 \times 10^{-4}$ \\
$\mathbf{4} \rightarrow \mathbf{5}$ & 14.07 & -5.96 & $1.48 \times 10^{1}$ \\
$\mathbf{4} \rightarrow \mathbf{6}$ & 7.42 & -6.73 & $7.58 \times 10^{5}$ \\
$\mathbf{4} \rightarrow \mathbf{9}$ & 27.63 & -5.51 & $2.11 \times 10^{-9}$ \\
$\mathbf{8} \rightarrow \mathbf{5}$ & 9.20 & -2.67 & $2.90 \times 10^{5}$ \\
$\mathbf{5} \rightarrow \mathbf{8}$ & 12.21 & -3.52 & $1.17 \times 10^{3}$ \\
$\mathbf{1 1} \rightarrow \mathbf{7}$ & 10.95 & -1.95 & $2.17 \times 10^{4}$ \\
$\mathbf{1 2} \rightarrow \mathbf{7}$ & 24.48 & -2.95 & $1.56 \times 10^{-6}$ \\
$\mathbf{7} \rightarrow \mathbf{1 1}$ & 16.80 & -1.66 & $1.28 \times 10^{0}$ \\
$\mathbf{7} \rightarrow \mathbf{1 2}$ & 33.94 & -0.10 & $7.55 \times 10^{-13}$ \\
$\mathbf{1 1} \rightarrow \mathbf{9}$ & 1.89 & -3.40 & $4.61 \times 10^{10}$ \\
$\mathbf{1 2} \rightarrow \mathbf{9}$ & 2.88 & -1.30 & $2.49 \times 10^{10}$ \\
$\mathbf{9} \rightarrow \mathbf{1 1}$ & 2.83 & -3.42 & $9.33 \times 10^{9}$ \\
$\mathbf{9} \rightarrow \mathbf{1 2}$ & 7.43 & -2.06 & $7.82 \times 10^{6}$ \\
& & &
\end{tabular}

30.68 to $49.50 \mathrm{kcal} / \mathrm{mol})$. We found five TSs showing this kind of interconversion $\left(\mathbf{T S}_{1-5}, \mathbf{T S}_{4-11^{*}}, \mathbf{T S} \mathbf{S}_{\mathbf{4 - 9}}, \mathbf{T S}_{7-12}\right.$, and $\left.\mathbf{T S} \mathbf{S}_{\mathbf{8}-11^{*}}\right)$.

$\mathbf{T S}_{7-11}$ connects a form possessing trans-trans-trans configuration with a form possessing trans-trans-cis configuration (Figure 8). In this case, due to the conformational restriction of the trans-trans-trans form, the conformational interconversion requires the movement of a greater number of atoms in comparison to the previous processess. Besides the movement of the $\mathrm{N}-\mathrm{H}$ group, this interconversion displays a concerted movement of the methylene and carbonyl groups of the peptide bonds which is not present in any of the previously discussed cases. We obtained only one TS showing this conformational interconversion with an energy gap of $24.42 \mathrm{kcal} / \mathrm{mol}$. Figure 8 gives the spatial view of the conformational interconversion type D.

TS $_{1-6}$ connects the global minimum with another trans - cis cis conformer. In this case, the movement is governed by the flipping of both the $\mathrm{N}-\mathrm{H}$ and the methylene groups. This movement involves a change of a cis-peptide bond plane, changing from cis-up to cis-down (Figure 9). The energetic requirement for this interconversion is $13.61 \mathrm{kcal} / \mathrm{mol}$. We found five TSs displaying this conformational interconversion, called type E $\left(\mathbf{T S}_{\mathbf{1 - 6}}, \mathbf{T S}_{\mathbf{2}-\mathbf{3}}, \mathbf{T S}_{\mathbf{4 - 8}}, \mathbf{T} \mathbf{S}_{\mathbf{5}-\mathbf{6}^{*}}\right.$, and $\left.\mathbf{T} \mathbf{S}_{\mathbf{9}} \mathbf{1 1}\right)$. It should be noted that the $\mathbf{T S}_{2-3}$ previously reported ${ }^{16}$ possessing 18.70 $\mathrm{kcal} / \mathrm{mol}$ corresponds to this type of process.

Interconversion of type $\mathrm{F}$ is closely related to that of type $\mathrm{E}$, but this process involves the change of the peptide bond crossing from cis to trans or vice versa (from trans to cis). $\mathbf{T S}_{3-5}$ (with $16.22 \mathrm{kcal} / \mathrm{mol}$, Figure 10), $\mathbf{T S}_{5-8}, \mathbf{T S}_{2-5}, \mathbf{T S}_{2-3}, \mathbf{T S} \mathbf{S}_{1-4}$, and $\mathbf{T S}_{1-10}$ show this kind of interconversion. It is interesting to note that, in this type of TSs, there are also TS structures displaying a significant movement of the methylene group located near the carbonyl group as a consequence of the conformational restriction. Thus, these TS types were referred to as $\mathrm{Fb}$. A particularly interesting TS structure of type $\mathrm{Fb}$ is $\mathbf{T S}_{1-2}$, which connects the global minimum with the lowest energy local minimum (crown form) within an energy gap of $12.49 \mathrm{kcal} / \mathrm{mol}$ (Figure 11). $\mathbf{T} \mathbf{S}_{\mathbf{1 - 9}}, \mathbf{T} \mathbf{S}_{\mathbf{1 - 1}}, \mathbf{T} \mathbf{T S}_{\mathbf{3}-\mathbf{6}}, \mathbf{T} \mathbf{S}_{\mathbf{4}-\mathbf{5}}$, and $\mathbf{T S}_{4-6}$ also show this kind of interconversion.

Vibrational frequencies for the optimized structures were computed to confirm the nature of the singular points along the PEHS. Thus, from the thermodynamic information $(\Delta H$ and $\Delta S)$ obtained in such an analysis, it was possible to perform an exploratory kinetic approach. It should be noted that each conformational interconversion corresponds to kinetics of first order. Considering the simplicity of this step, it is possible to calculate the specific constant of velocity for each conformational interconversion using the Eyring formulation.

$$
k=\frac{R T}{N_{\mathrm{A}} h} \mathrm{e}^{\Delta S / R} \mathrm{e}^{-\Delta H^{\circ} / R T}
$$


These results are summarized in Table 4, last column. For these simulations, we assume that each conformer possesses the same amount in the starting time. Our results indicate that the interconversions for the high-energy conformers are practically instantaneous, remaining an equilibrium mixture of only conformers $\mathbf{1}$ and 2 with a minimal contribution of conformer $\mathbf{3}$. The relationship at the equilibrium time is $55.3 \%$ for conformer $\mathbf{1}$, $34.4 \%$ for conformer $\mathbf{2}, 1.4 \%$ for conformer 3 , and the rest for the other conformers. These results were obtained from RHF/ 6-31G(d) calculations; however, all of the calculations reported here are coincident, predicting an equilibrium mixture between conformers $\mathbf{1}$ and $\mathbf{2}$ at room temperature.

\section{Conclusions}

The PEHS of cyclic Gly-Gly-Gly was investigated using theoretical calculations. Ab initio and DFT computations provide a clear picture for the conformational PEHS of this molecule from both the structural and energetic points of view.

The exploration of a conformational energy hypersurface by methods that ignore all features other than local energy minima does not always provide a satisfactory picture of the problem. Thus, it is very desirable to determine, at least, the lowest energy transition states linking all pairs of conformations. The elementary steps in conformational interconversion paths for cyclic triglycine were here discussed in detail. The different conformational interconversions were analyzed to offer a clearer understanding of the conformational intricacies of the PEHSs of cyclic tryglicine. Altogether, 39 geometries (12 minima and 27 transition states) were found to be important for a description of the conformational features of CTG. Our results indicate that two forms, a trans-cis-cis and the cis-cis-cis "crown" form, contribute significantly to the equilibrium mixture at room temperature. RHF/6-31G(d) predicts that the conformational interconversion between the crown and twist forms requires $12.49 \mathrm{kcal} / \mathrm{mol}$.

An exploratory topological analysis of the PEHSs is also reported here. This preliminary analysis is very simple and schematic; however, it provides a logical explanation for the conformational intricacies of these cyclic compounds, a feature that is difficult to rationalize without a model that allows visualization of the overall conformational problem.

Acknowledgment. Grants from Universidad Nacional de San Luis (UNSL) supported this work. R.D.E. is a member of the
Consejo Nacional de Investigaciones Científicas y Técnicas (CONICET-Argentina) staff.

\section{References and Notes}

(1) Spatola, A. F. In Chemistry and Biochemistry of Amino Acids, Peptides and Proteins; Weinsteing, B., Ed.; Marcel Dekker: New York, 1983; Vol. VII, p 267.

(2) Dygort, M.; Go., N.; Scheraga, H. Macromolecules 1975, 8, 750.

(3) Lipton, M.; Still, W. J. Comput. Chem. 1988, 9, 343.

(4) Suvire, F.; Santágata, L.; Bombasaro, J.; Enriz, R. J. Comput. Chem. 2006, $27,188$.

(5) Groth, P. Acta Chem. Scand. 1976, A30, 838.

(6) Dale, J.; Titlestad, K. J. Chem. Soc. D 1969, 12, 656.

(7) Titlestad, K. Acta Chem. Scand. B 1975, 29, 153.

(8) Malthête., J.; Collet, A. Nouv. J. Chim. 1985, 9, 151.

(9) Hioki, H.; Kinami, H.; Yoshida, A.; Kojima, A.; Kodama, M.; Takaoka, S.; Veda, K.; Katsu, T. Tetrahedron Lett. 2004, 45, 1091.

(10) Kessler, H.; Bermel, W.; Krack, G.; Bats, J.; Fuess, H.; Hull, H. Chem. Ber. 1983, 116, 3164.

(11) Kessler, H. Angew. Chem., Int. Ed. Engl. 1982, 21, 512.

(12) Bats, J.; Fuess, H. Acta Crystallogr. 1982, B38, 1004.

(13) Bats, J.; Fuess, H. J. Am. Chem. Soc. 1980, 102, 2065.

(14) Kessler, H.; Schuck, R.; Siegmeier, R.; Bats, J.; Fuess, H.; Foster, H. Liebigs Ann. Chem. 1983, 231.

(15) Ramakrishnan, C.; Paul, P.; Ramnarayan, K. Proc. Int. Symp. Biomol. Struct, Interactions, Suppl. J. Biosci. 1985, 8, 239.

(16) Zamora, M.; Suvire, F.; Enriz, R. J. Comput. Chem. 2008, 29, 280.

(17) Frisch, M. J.; Trucks, G. W.; Schlegel, H. B.; Scuseria, G. E.; Robb, M. A.; Cheeseman, J. R.; Montgomery, J. A., Jr.; Vreven, T.; Kudin, K. N.; Burant, J. C.; Millam, J. M.; Iyengar, S. S.; Tomasi, J.; Barone, V.; Mennucci, B.; Cossi, M.; Scalmani, G.; Rega, N.; Petersson, G. A.; Nakatsuji, H.; Hada, M.; Ehara, M.; Toyota, K.; Fukuda, R.; Hasegawa, J.; Ishida, M.; Nakajima, T.; Honda, Y.; Kitao, O.; Nakai, H.; Klene, M.; Li, X.; Knox, J. E.; Hratchian, H. P.; Cross, J. B.; Adamo, C.; Jaramillo, J.; Gomperts, R.; Stratmann, R. E.; Yazyev, O.; Austin, A. J.; Cammi, R.; Pomelli, C.; Ochterski, J. W.; Ayala, P. Y.; Morokuma, K.; Voth, G. A.; Salvador, P.; Dannenberg, J. J.; Zakrzewski, V. G.; Dapprich, S.; Daniels, A. D.; Strain, M. C.; Farkas, O.; Malick, D. K.; Rabuck, A. D.; Raghavachari, K.; Foresman, J. B.; Ortiz, J. V.; Cui, Q.; Baboul, A. G.; Clifford, S.; Cioslowski, J.; Stefanov, B. B.; Liu, G.; Liashenko, A.; Piskorz, P.; Komaromi, I.; Martin, R. L.; Fox, D. J.; Keith, T.; Al-Laham, M. A.; Peng, C. Y.; Nanayakkara, A.; Challacombe, M.; Gill, P. M. W.; Johnson, B.; Chen, W.; Wong, M. W.; Gonzalez, C.; Pople, J. A. Gaussian 03, revision B.05; Gaussian, Inc.: Pittsburgh, PA, 2003.

(18) Santagata, L.; Suvire, F.; Enriz, R.; Torday, J.; Csizmadia, I. THEOCHEM 1999, 33, 465.

(19) Santagata, L.; Suvire, F.; Enriz, R. THEOCHEM 2000, 89, 507.

(20) Santagata, L.; Suvire, F.; Enriz, R. THEOCHEM 2001, 173, 536.

(21) Santagata, L.; Suvire, F.; Enriz, R. THEOCHEM 2001, 91, 571.

(22) Gonzalez, C.; Schlegel, H. J. Chem. Phys. 1989, 90, 2154.

(23) Gonzalez, C.; Schlegel, H. J. Phys. Chem. 1990, 94, 5523.

\section{JP905187K}

